\title{
Telemedicine: Underutilized Tool of Global Health
}

Challenges bring an opportunity for success and each success is followed by a new challenge. Challenges and successes witnessed in the discipline of medicine are numerous. When world was preparing to enter the new millennium twenty years ago many great challenges were faced by medical professionals in-order to implement plans of quality health provision to every human globally. "Health for all" strategy, Millennium Development Goals (MDGs) and many other similar plans are the examples of such great vision to provide health care with equitable distribution, appropriate technology, community participation and acceptance among masses.

Telemedicine is the use of telecommunication and information technologies in order to provide clinical health care at far flung areas with connections through networking. In absence of telemedicine, the equitable access to deliver better health care is difficult especially in remote communities as some health standards could not be implemented on providers and recipients in different places at the same time. Recent advances in telemedicine have created opportunities for health care providers and recipients to overcome the barriers of lack of awareness and deficiency of standard approach towards prevention and management. The experts could extend their teaching and evidence based medical practices in order to increase standards of health care globally. Information technology helps to eliminate distance and communication barriers and can improve access to medical services that are deficient or in distant rural communities. The communities in farflung areas and isolated populations can receive health care from specialist residing at a distance without patient to the need of travel to visit them.

Telemedicine can also be used to train health workers in field, providing evidence based medical practices, facilitation in standard operating procedures, delivery of medical education, sharing best practices and experiences. Recent advances in mobile technology and the potential for global health elevated telemedicine as a popular force in health care. The special- ties that can be focused during implementation of telemedicine practices include preventive medicine, radiology, pathology, cardiology, mental health services, primary care, emergency care, physical rehabilitation, pediatrics, obstetrics, immunization, nutrition, mother and child health, disaster management, epidemic control, health system monitoring and management and many others.

Telemedicine in developing countries like Pakistan is a tool of global health. Pakistan is an over populated country where people are, by the large, deprived of proper medical facilities especially those living in far flung areas. The implementation of telemedicine can facilitate the patients and educate the health care providers for the purpose of improving patient care. In Pakistan, bio-metric verification by Pakistan Telecommunication Authority (PTA) has declared mobile phone users in the country; according to which the figure for mobile phone users is estimated to be near 114.7 million. Thus big resource is available that can be channelized to improve health and prevent disease through telemedicine across the country and beyond geographical boundaries.

According to World Health Organization (WHO), the use of telemedicine in provision of health care is not simply about technology, but a means to reach an outcome, such as better decisions for provision of prevention and management, vulnerable populations received high quality and safer care, more awareness about health making people to have better choices for their health, better surveillance of communicable and non-communicable disease, government become more responsible and aware of health needs of populations. Moreover equitable, appropriate, acceptable, effective and efficient monitoring of resources at Healthcare Centers.

The satisfaction of patients and health care providers were found positive over time. According to Care Hill more than $80 \%$ of consumers find their health care to be satisfactory. Telemedicine is occasionally blamed of being driven by commercial rather than public

1 ANNALS VOL 22, ISSUE 1, JAN. - MAR. 2016 
health interests. However in spite of this criticism, telemedicine is linking the rich communities with poors globally.

Nevertheless, telemedicine is an effective tool for surveillance, monitoring, standardization, research, and implementation. There is no alternative to tele- medicine. There are several telemedicine networks linking the industrialized and developing world, this is an area that is likely to grow in the future. Global health strategies have to focus on underutilized tool for better access to health care.

Dr. Saira Afzal 